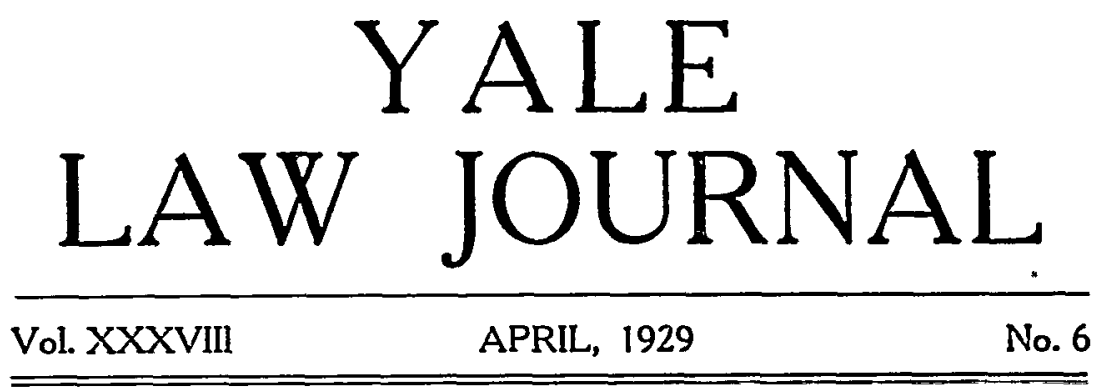

\title{
AN INSTITUTIONAL APPROACH TO THE LAW OF COMMERCIAL BANKING
}

\section{UNDERHILl MOORE AND THEODORE S. HOPE, JR.}

The central problem of the lawyer is the prediction of judicial and administrative decisions of government officers. For its solution he has looked to the cases recording the decisions of such officers in the past, and has made no systematic study of other data. In adopting this course, he has postulated that study of the relation between the decisions and "the facts" of recorded cases would be sufficient to enable him to formulate laws, from which he could predict future judicial and administrative behavior. His method has been in part induction, in part hypothesis and verification. He has compared the cases and attempted to classify them, in accord with similarities and dissimilarities in their facts, to abstract the common element of each class, and to correlate this common element with the decisions. Finally, the results of such correlations have been generalized into laws of judicial and administrative behavior, from which it is supposed that, if "the facts of the case" are known, future behavior" of a particular judge in a particular case may be predicted.

This supposition has not been verified in experience. In a case of any novelty whatever, the lawyer in predicting judicial behavior is compelled to rely not on logical application of those laws but on an intuitional judgment based on an experience only a small part of which is those laws. In part, the character of the problem accounts for his predicament. The problem is 
to predict the judicial behavior of a particular judge at a particular time and place in an uncontrolled environment. If the judicial behavior of every judge in the past had been correlated with every significant element in the situation including his behavior, and had the procedure netted very precise applicable laws, yet a prediction as probable as that of the physicist predicting the result of a time-worn experiment would not be possible. For in the experiment every element not present in the prior observations is painstakingly excluded. .Such exclusion is not possible in the actual behavior situations of every-dry life, including that in which the particular judge is to act. This difficulty of the lawyer, it should be noted, is not the result of his not employing an experimental technique. If it were possible to observe judicial behavior under conditions which excluded all elements not at the time under observation, by the experimental method of natural science, and as a result formulate laws of judicial behavior, the lawyer would certainly be no more able to predict the reaction of a particular judge in a particular situation not under experimental control than the natural scientist is able to predict whether a particular wild perennial will bloom next year. ${ }^{\mathrm{I}}$

In greater part, however, the lawyer's limited success in formulating laws of judicial behavior is probably to be accounted for by his failure to attempt to correlate judicial behavior with any events except the "facts of the case." His persistent pursuit of his laws in the "facts of the case" may be explained by the fact that the judges and administrators themselves in their opinions began quite irrelevantly and ambiguously to say that their behavior was the necessary consequence of these laws. From necessary logical deduction to necessary behavior was an easy step, and the transformation of scientific generalizations into Law was complete. Whether this new and puissant being was the daughter of God, of nature, or of the state, her will had been ascertained by correlating judicial behavior with the facts of the case, and should therefore continue to be so ascertained.

There are innumerable other facts with which official behavior

1 "The scientist is able to determine a limited number of laws-that under given conditions given results will invariably follow. He is not, however,

- able to give a complete causal explanation of any phenomenon. He cannot, for example, explain completely why a certain wild rose bloomed undor a certain hedge at a certain moment. In order to do this it would be necessary to begin with the formation of the material world, determine every force and measure every influence in the universe in the order of thoir reciprocal actions down to the present moment.

"The complete determination of the causation of any act of human bohavior would be a task not less impossible than this." THOMAS AND Thomas, The ChInd in America (1928) 553. 
might be correlated. The lawyer's colleagues in the other sciences of human behavior have attempted to correlate behavior with "racial" traits, structure, physiological processes, psychological adjustment, "personality," intelligence, and co-existent culture. The lawyer's single-mindedness has saved us thus far from a racial jurisprudence, a neurotic jurisprudence, etc., but it has unfortunately deprived him of worthwhile scientific experience. It is proposed that he begin acquiring that experience.

It is proposed that the field of the lawrer's attention be extended to include not only the relation between judicial behavior and "the facts of the case," but also the relation between judicial behavior and institutional (frequent, repeated, usual) ways of behaving (e.g., doing business) in the contemporary culture of the place where the facts happened and the decision was made. If such a relation is found to be significant, a step towards more reliable prediction will have been made. The step will have been a short step, however. That is found to be true of the culture and decisions of today may not be true of the changed culture of tomorrow. What is found to be true of the group of cases examined may not be true of any other group of cases.

"The facts" of the recorded cases classified in the traditional "legal" categories are a small, and very probably non-representative, sample of all behavior, atypical as well as typical. The cases are distinguished by dissimilarity rather than similarity one to another. Thus in order that the "legal" categories might be multitudinous of individual cases, it was necessary that their differentiating concepts be exclusive of few individual cases. In consequence, the "legal" categories are inadequate for classification, and the "legal" abstractions (which were formulated with reference to those categories) are inadequate for manipulation of conduct typical and atypical. In a study such as that proposed, therefore, it will unfortunately be necessary to disregard the "laws of judicial behavior" stated in opinions since they are formulated in terms of "legal" categories. For a like reason it will also be necessary, in a study such as that proposed, to employ a method of procedure in detail quite dissimilar to the lawyers' traditional method of procedure. There follows an elaboration of such a methodology.

The subject of the proposed study is human behavior. The study proposed is a systematic study. A necessary step in such a study is the differentiation and organization of fields of investigation-the choice and logical elaboration of categories and 
sub-categories. The behavior constituting any such field includes only one aspect of mere fragments of observed behavior. This choice of a single aspect is inescapably the process of classification. The process of classification includes the selection of organizing common factors. The field of investigation and its subdivisions are defined by the organizing common factors selected.

The object of the proposed study is the relation between the decision in a particular case and the type and degree of deviation of the behavior followed by that decision ("the facts in the case") from institutional behavior. A necessary step is comparison of the behavior followed by the decision with institutional behavior. The making of such a comparison necessitates the formulation of abstractions through the manipulation of which comparison may be made. This formulation is independent of observation within any field of investigation; the abstractions formulated presuppose the definition and selection of some field of investigation, but not the definition and selection of any particular field of investigation.

The subject-matter of any field of investigation may be considered as a class of entities possessing the common factors by which the field is defined. The subject-matter of a field of investigation whose subject-matter is behavior may be considered as a class of segments of behavior possessing the common factors by which the field is defined. A segment of behavior is a succession of events of behavior. A segment of behavior which is an entity is a succession of events of behavior in some relation with each other. The relation chosen to determine the events constituting such a segment of human behavior might have been, but was not, solely a space-time relation (e.g., all events occurring today in this room). The relation chosen was rather that often crudely referred to as the relation of cause and effect, or the causal relation. ${ }^{2}$ The greatest segment of human behavior in causal relation and possessing the common factors by which the field is defined will be referred to as a transaction-series. The least segment of behavior in causal relation and possessing the

2 A particular event $E$ may be said to be in causal relation with another particular event $e$ if the likelihood that event $E$ will be succeeded by ovents $e_{,} e_{1}, e_{2}, e_{3}$, etc., at particular intervals of space and time is greater than the likelihood that it will be succeeded by other particular events.

The likelihood referred to is not an a priori mathematical probability. That cannot be computed since the equally likely cases cannot bo enumerated. It is not an a posteriori statistical probability. That has not beon observed. It cannot too strongly be intimated that the judgment as to likelihood which is here supposed is a qualitative subjective judgment, and not a quantitative objective judgment. The word likelihood is used in this same sense hereafter. It refers neither to the result of a mathomatical inference nor to the result of a statistical observation. 
common factors by which the field is defined will be referred to as a transaction. A transaction-series is obviously a succession of one or more transactions in causal relations with each other.

A less segment of behavior than a transaction, because more concise than a transaction, might be, for some purposes, more conveniently dealt with than a transaction. One such segment of behavior, which will be referred to as a tcrm in a transaction, is defined as follows: a term in a transaction is that segment of behavior in a transaction, during which one at least of the individuals, who was an actor in the transaction at the commencement of the term, continues (without the intervention of another actor) to be an actor in the transaction. A transaction is obviously a succession of one or more terms in causal relation.

Classes of transactions may also be organized with reference to descriptive similarity (looking alike, rather than having like consequences). Such classes are defined by a generalized description of the transactions of which the class is composed. An hypothetical transaction corresponding point for point with such a generalized description is, if the class of transactions which is defined by that description is sufficiently large, referred to as a sequence. An actual transaction which would, if hypothetical, be a sequence, is referred to as a sequcntial transaction. An actual transaction-series constituted of sequential transactions is referred to as a sequential transaction-serics.

Institutional behavior (i.e., behavior which frequently, repeatedly, usually occurs) may thus be defined as a class of sequential transactions. Actually, transactions are preceded, accompanied, and followed by other transactions. Some sequential transactions may frequently succeed certain series of sequential transactions. The relation of frequently followingfrequently preceding is referred to as the institutional relation. The class of transactions which satisfies the condition of being sequential transactions in institutional relation is referred to as the institution. ${ }^{3}$ Sequences in institutional relation are referred to at as institutional sequenecs.

The institutional analogue to a transaction-series is referred to as a sequence-series.

Any actual transaction which does not satisfy the condition of being a sequential transaction in institutional relation is referred to as a deviational transaction.

The problem of comparison may now be restated as a problem

3 Within any particular field of observed behavior the institution is found, as well as may be, by the most available means for ethnologizing a particular present day culture: interviews and observation, questionnaires, letters, reports, books, and insight. 
of comparing with comparable sequence-series actual transaction-series followed by judicial behavior (i.e., in causal relation with succeeding judicial behavior). It is apparent that the initial deviational transaction and succeeding transactionswhich by definition are also deviational-may in any transaction-series number one, two, three, etc. It will be convenient to formulate first a method of comparison of a transaction-series which is concluded by an initial deviational transaction.

That fragment of a transaction-series which precedes the initial deviation is most comparable, since it is institutional, with an identical fragment of a sequence-series. The entire transaction-series is thus to be compared with the institutional fragment of itself plus a sequence in institutional relation therewith. That comparison will be made transaction by transaction, since it is inconvenient (if not impossible) to compare a transaction-series with a sequence-series. But such comparison need only be made between a deviational transaction and its comparable sequence. It is necessary to select a basis for choice of such a particular sequence.

An actual transaction, it will be recalled, which does not satisfy the condition of being a sequential transaction in institutional relation, is a deviational transaction. A transaction has been defined as the least segment of behavior in causal relation and possessing the common factors by which the field is defined. The deviational transaction is comparable to any sequence possessing any one of the common factors. If the deviational transaction possesses more than one common factor the sequence chosen as comparable depends upon its possessing the common factor differentiating the subdivision of the field within which the transaction for the time being is under observation.

It will now be possible to formulate a method of comparison of a transaction-series which includes two deviational transactions.

The rule for choice of a sequence comparable to any initial deviational transaction is identically the rule for choice of a sequence comparable to a single deviational transaction. The rule for choice of a sequence comparable to a second deviational transaction is in part identically the rule for choice of a sequence comparable to any initial deviational comparison. One comparison is made with a sequence chosen in accordance with that rule. A second comparison is also made. This second comparison is made with a sequence in institutional relation with the sequence comparable to the initial deviational transaction preceded by the institutional fragment of the transaction-series. In making this comparison the sequence comparable to the initial devia- 
tional transaction is substituted for that transaction in the transaction-series. ${ }^{1}$

The method of comparison of an initial or any succeeding deviational transaction with its comparable sequence is to observe (1) the degree by which the prior terms in the deviational transaction deviate from the correlative terms in the comparable sequence, and (2) the degree by which the concluding terms in the deviational transaction deviate from the correlative terms of the comparable sequence.

The degree by which the prior terms in the deviational transaction deviate from the correlative terms of the comparable sequence is observed by supposing the concluding terms of the deviational transaction to be the same as the concluding terms of the comparable sequence. In this way it is hoped that it will be possible to compare a deviational transaction and its comparable sequence as possibly alternative devices for obtaining the same result.

The degree by which the concluding terms in the deviational transaction deviate from the correlative terms of the comparable sequence is observed by supposing the prior terms in the deviational transaction to be the same as the correlative terms in the comparable sequence. By this assumption it is intended to confine this second comparison to a comparison of ends, in the same way as the first comparison was confined to a comparison of means.

\section{II}

It is now pertinent to define the particular field of investigation in which it is proposed that this method should be applied, and exemplify its application in that field. The field can best be defined by stating the common factors which differentiate from other behavior the behavior constituting the field of investigation, and describing the behavior so differentiated.

Implicit in the choice of the common factors is the concept of

1 This rule for comparison of a second deviational traneaction with two possibly different sequences results from an hypothesis that it is to the highest degree likely that the judicial behavior following the tranzactionseries will be either the same as it would have been if instcad of the initial deviational transaction an institutional transaction had occurred; or the same as it would have been if instead of the initial deviational transaction nothing had occurred; and that whether it is the one or the other may depend in large measure upon the degree of deviation of the initial deviational transaction.

An obvious extension of this hypothesis to the case of a third deviational transaction requires comparison of such a transaction with four poscibly different sequences; of an $n^{\text {th }}$ deviational transaction with $2^{7=1}$ possibly different sequences. Fortunately all the possibilities of difference are seldom realized. 
deposit currency. This concept is derived from certain transactions between a commercial bank and some other individual or group of individuals.

Deposit currency is but one among many forms of commercinl bank credit; it is credit on the current checking account. Deposit currency of a particular bank in favor of a particular customer exists in the amount of the balance of the current checking account of that customer with that bank. The term balance is here used in a special sense, to refer to the amount of deposit currency of a particular customer. The balance may be described as the limiting amount up to which checks are likely to be drawn by the customer and honored by the bank. This description is obviously incomplete; nevertheless, it sufficiently approximates a description which to be exhaustive would of necessity enumerate many other sorts of behavior.

Deposit currency of a particular customer is created when the balance of that customer increases. Deposit currency of a particular customer is extinguished when the balance of that customer decreases. Deposit currency of a particular customer is transferred when the balance of that customer decreases and the balance of another customer (the transferee) of the same or another bank increases. Accounts between, for example, buyers and sellers are liquidated when it becomes unlikely that thereafter payment will be either demanded or offered.

The field of investigation is constituted of transactions which have either resulted in the creation of deposit currency, the extinguishment of deposit currency, the transfer of deposit currency, the liquidation of accounts by means of transfer of deposit currency, or which have created a likelihood that one or another such result would follow but, without any such result, have terminated by its becoming no longer likely.

The field of investigation is divided into four main subdivisions. A transaction may fall within more than one subdivision. As between some subdivisions this is obviously true; it could readily be shown to be true as among any subdivisions. If a particular transaction falls in more than one subdivision, in each of those subdivisions particular attention is paid only to that aspect of the transaction which brings it within that subdivision.

(a) The first subdivision includes transactions which have resulted in the creation of deposit currency, or which have created a likelihood that deposit currency would be created but, without its being created, have terminated by its becoming no longer likely that deposit currency would be created. .

(b) The second subdivision includes transactions which have resulted in the extinguishment of deposit currency, or which have created a likelihood that deposit currency would be 
extinguished but, without its being extinguished, have terminated by its becoming no longer likely that deposit currency would be extinguished.

(c) The third subdivision includes transactions which have resulted in the transfer of deposit currency, or which have created a likelihood that deposit currency would be transferred but, without its being so transferred, have terminated by its becoming no longer likely that deposit currency would be transferred.

(d) The fourth subdivision includes transactions which have resulted in the liquidation of accounts by means of the transfer of deposit currency, or which have created a likelihood that accounts would so be liquidated but, without their being so liquidated, have terminated by its becoming no longer likely that accounts would be so liquidated.

Application of the method of comparison will be exemplified for some of these subdivisions.

Consider the following transaction-series:

(1) Let it be assumed that the $A$ corporation, a customer of the $B$ bank, has a balance of $\$ 10,000$ with that bank." Behavior occurs on the part of appropriate officers of the customer and appropriate bank officials which may be synopsised as follows: the $A$ corporation and the $B$ bank agree that upon the happening of a named contingency the $A$ corporation shall offer and the $B$ bank accept for discount at six per cent the $A$ corporation's ninetyday note for $\$ 10,000$ secured by mortgage on its fixtures and stock-in-trade, and that its account shall thereupon be credited with the proceeds of the discount. Upon the happening of the contingency the $A$ corporation tenders its note and mortgage; the bank rejects the tender and the $A$ corporation is compelled to borrow in the open market at the then market rate of nine per cent. The $A$ corporation now sues the bank for $\$ 75$, threefourths of one per cent of $\$ 10,000$.

(2) Let it be assumed that $A$, a customer of the $E$ bank, has a balance of $\$ 10,000$ with that bank. Behavior occurs on the part of the customer and appropriate bank officials which may be synopsised as follows: $A$ signs ten instruments in form promissory notes payable to the order of the $E$ bank, each in the amount of $\$ 1,000$, with the space for the date left blank, and hands them to the appropriate bank officials; it is agreed that if a check of $A$ is presented for payment for an amount in

\footnotetext{
5 This form of statement is obviously elliptical. When it is said that $A$ 's balance is $\$ 10,000$, nothing is said about the order or amount of the past transactions between $A$ and his bank. What is said is that whatever they may have been they have been in institutional relation with each other, and that the drawing and honoring of a check for $\$ 10,000$ would be in institutional relation with the last of them.
} 
excess of his then balance as it appears on the books of the bank, the check shall not be dishonored, but the then date shall be inserted in one of these notes, it shall forthwith be discounted at the rate of six per cent, and the proceeds credited to $A$ 's account. $A$ then draws his check for $\$ 10,500$ payable to $C$ 's order and hands it to $C$, who indorses it and deposits it to the credit of his account with the $D$ bank of which he is a customer. The $D$ bank presents the check to the $B$ bank through the clearing-house. The $B$ bank sends it back marked "insufficient funds." $A$ now sues the bank for damages for the dishonor of this check.

(3) Let it be assumed that $A$, a customer of the $B$ bank, has a balance of $\$ 10,000$ with that bank. At the bank offices and during banking hours he orally requests of the paying teller that he pay him the sum of $\$ 500$. The paying teller refuses to comply with the request, and $A$ sues the $B$ bank for the amount of $\$ 500$.

(4) Let it be assumed that $A$, a customer of the $B$ bank, has a balance of $\$ 10,000$ with that bank. He draws his check for $\$ 1,000$ payable to the order of $C$, who indorses it and deposits it to the credit of his account with the $D$ bank, of which he is a customer. The $D$ bank presents the check to the $B$ bank through the clearing house. The $B$ bank sends it back marked "insufficient funds." The $D$ bank now sues the $B$ bank for $\$ 1,000$, the amount of the check.

As to each of these transaction-series, it has already been assumed that that part of the series symbolized in the words "Balance $\$ 10,000$ " has been institutional. As to each of these series let it also be assumed that succeeding transactions are deviational.

In making the comparison, the following notational system will be employed:

Double lines ( $\|, / /)$ to indicate the end of a transaction or sequence. Vertical double lines $(\|)$ to indicate the end of a sequential transaction in institutional relation, or of an institt1tional sequence. Oblique double lines $(/ /)$ to indicate the end of a deviational transaction. A vertical single line $(\mid)$ to indicate the end of a term in a transaction or sequence.

(1) The assumed transaction-series numbered (1) may, in symbolic notation, be expressed as follows:

TBal. $\$ 10,000||$ Agreement to lend $\$ 10,000 \mid$ Note for $\$ 10,000$ offered for discount | Rejected //

It will be observed that the series is concluded by an initial deviational transaction. That transaction is within the field of investigation, and within the first main subdivision of that 
field (the "creation subdivision") ; initially, it became likely that deposit currency would be created by subsequent discount of the customer's note; when the note was refused to be discounted that likelihood ceased.

The transaction, then, is comparable to a sequence in institutional relation with the transactions symbolized by "Balance $\$ 10,000$ " and chosen from the "creation" subdivision of the field of investigation. There may be many such sequences. Among them may be, for example, the deposit of currency, the offering for discount of the customer's note and its refusal, the offering for discount of the customer's note and its acceptance for discount. If these be assumed to be such sequences, and it be further assumed that "agreements to lend" such as the one assumed for this transaction-series, performed as well as unperformed, are in this particular community deviational, the particular sequence for comparison may be chosen from among these three sequences. The particular sequence chosen for comparison is the last of these three sequences. Implicit in this choice is the judgment that the sequence chosen is more similar to the transaction than other possible sequences; in other words, that the transaction to be compared is most nearly identical with the transactions typified by the sequence chosen for comparison in respect of likely following transactions.

It will be recalled that the first comparison is made by substituting for the concluding terms of the transaction the correlative terms of the comparable sequence. The transaction, so modified, and its comparable sequence are, in symbolic notation, expressed as follows:

IA $\left\{\begin{array}{c}\text { TBal. } \$ 10,000 \text { || Agreement to lend } \$ 10,000 \text { | Note for } \$ 10,000 \\ \text { offered for discount | Accepted // } \\ \text { sBal. \$10,000 II Note for } \$ 10,000 \text { offered for discount I } \\ \text { Accepted || }\end{array}\right.$

The degree of deviation should be measured in accordiance with the rules hereafter stated.

It will also be recalled that second comparison is made by substituting for the prior terms of the transaction the correlative terms of the comparable sequence. The transaction, so modified, and the comparable sequence are, in symbolic notation, expressed as follows:

IB $\left.\right|_{\text {SBal. } \$ 10,000}||$ Note for $\$ 10,000$ offered for discount | Aeccptsd || \{TBl. $\$ 10,000$ || Note for $\$ 10,000$ offered for discount | Rejeetcd //

No zules for measurement of the degree of deviation of the concluding terms of a deviational transaction from the correlative terms of its comparable sequence have as yet been formulated. The concluding terms of the deviational transactions in 
this and the succeeding exemplary comparisons seem to be either identical with, or logically opposite to, the correlative terms of the comparable sequence. Apparently the statement that they are either identical or logically opposite is itself a measurement of the degree of deviation.

(2) The assumed transaction-series numbered (2) may, in symbolic notation, be expressed as follows:

TBal. $\$ 10,000$ || Offer of "overdraft agreement" for $\$ 10,000$ with notes of customer | Accepted // Check for $\$ 10,500$ to payee | Payce to his bank | Presented through clearing-house | Dishonored //

It will be observed that the initial deviational transaction is followed by a second deviational transaction.

The initial deviational transaction is within the "creation" subdivision of the field of investigation. It is comparable to the sequence which is expressed in symbolic notation as follows:

sBal. $\$ 10,000$ || Note for $\$ 10,000$ offered for discount | Accepted ||

It will be recalled that two comparisons of a transaction with a comparable sequence are made.

The first comparison of the initial deviational transaction is made by substituting for the concluding terms of the transaction the correlative terms of the comparable sequence. The transaction, in this instance, remains unmodified after such substitution. The transaction and its comparable sequence are, in symbolic. notation, expressed as follows:

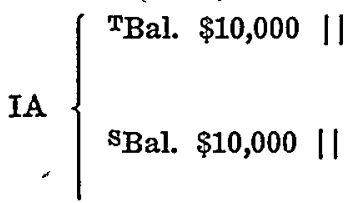
Offer of "overdraft Note for $\$ 10,000$ of- fered for discount agreement" for $\$ 10,000$ with notes of customer
| Accopted //
| Accopted ||

The degree of deviation should be measured in accordance with the rules hereafter stated.

The second comparison of the initial deviational transaction is made by substituting for the prior terms of the transaction, the correlative terms of the comparable sequence. The transaction, so modified, and the comparable sequence are, in symbolic notation expressed as follows:

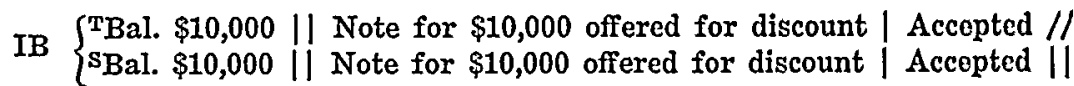

The second deviational transaction is to be compared successively with two comparable sequences. It will be recalled that the first comparison was to be made with a sequence chosen by identically the rule by which a sequence comparable to an initial 
deviation is chosen. The comparable sequence, chosen by that rule, is, in symbolic notation:

sBal. $\$ 10,0000$ || Check for | Payee to | Presented through | Paid || $\$ 10,000$ to his bank clearing-house payee

The first comparison of the second deviational transaction with its first comparable sequence is made by substituting for the concluding terms of the transaction the correlative terms of the comparable sequence. The transaction, so modified, and its comparable sequence are, in symbolic notation, expressed as follows:

II A $\left\{\begin{array}{c}\text { TBal. } \$ 10,000 \text { || } \begin{array}{c}\text { Check for | Payee to | Presented through | Paid || } \\ \$ 10,500 \text { to his bank clearing-house } \\ \text { payee }\end{array} \\ \text { SBal. } \$ 10,000 \text { || } \begin{array}{c}\text { Check for | Payee to | Presented through | Paid || } \\ \$ 10,000 \text { to his bank clearing-house } \\ \text { payee }\end{array}\end{array}\right.$

The degree of deviation should be measured in accordance with the rules hereafter stated.

The second comparison of the second deviational transaction with its first comparable sequence is made by substituting for the prior terms of the transaction the correlative terms of the comparable sequence. The transaction, so modified, and its comparable sequence are, in symbolic notation, expressed as follows:

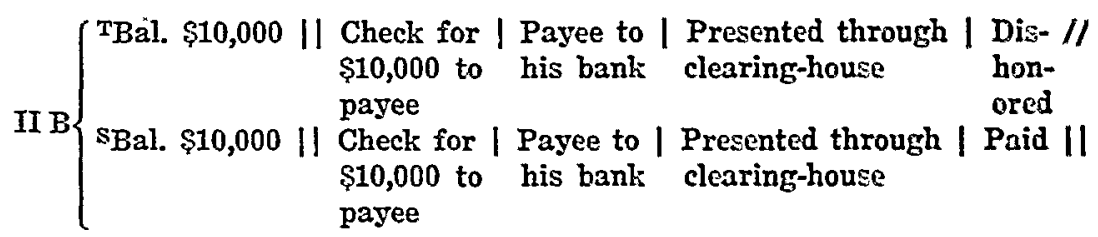

The second deviational transaction is to be compared, also, with a sequence in institutional relation with the sequence comparable to the initial deviational transaction preceded by "Bal. $\$ 10,000$." The comparison is to be made by substituting in the transaction-series its comparable sequence for the initial deviational transaction. In symbolic notation, the transaction-series, so modified, and its comparable sequence-series may be expressed as follows:

\footnotetext{
TBal. $\$ 10,000$ || Note for $\$ 10,000$ offered for discount | Accepted // II C clearing-house | Dishonored //

SBal. $\$ 10,000||$ Note for $\$ 10,000$ offered for discount | Accepted || Check for $\$ 10,500$ to payee | Payee to his bank| Presented through clearing-house | Paid ||
} 
The first comparison of the second deviational transaction with its second comparable sequence is made by substituting for the concluding terms of the transaction the correlative terms of the comparable sequence. The transaction, so modified, and its comparable sequence are, in symbolic notation, expressed as follows:

III A $\left\{\begin{array}{c}\text { TBal. } \$ 10,000 \text { || Note for } \$ 10,000 \text { offered for discount | Accepted // } \\ \text { Check for } \$ 10,500 \text { to payee | Payee to his bank | Presented } \\ \text { through clearing-house | Paid // } \\ \text { sBal. } \$ 10,000 \text { || Note for } \$ 10,000 \text { offered for discount | Accepted || } \\ \text { Check for } \$ 10,500 \text { to payee | Payee to his bank | Presented } \\ \text { through clearing-house | Paid || }\end{array}\right.$

Obviously the terms to be compared are identical. A measurement of the degree of deviation is implicit in this statement.

The second comparison of the second deviational transaction with its second comparable sequence is made by substituting for the prior terms of the transaction the correlative terms of the comparable sequence. The transaction, in this instance, remains unmodified after such substitution. The transaction and its comparable sequence are, in symbolic notation, expressed as follows:

III B $\left\{\begin{array}{l}\text { TBal. } \$ 10,000 \text { || Note for } \$ 10,000 \text { offered for discount | Accepted // } \\ \text { Check for } \$ 10,500 \text { to payee | Payee to his bank | Presented } \\ \text { through clearing-house | Dishonored // } \\ \text { sBal. } \$ 10,000 \text { || Note for } \$ 10,000 \text { offered for discount | Accepted || } \\ \text { Check for } \$ 10,500 \text { to payee | Payee to his bank | Presented } \\ \text { through clearing-house | Paid || }\end{array}\right.$

(3) The assumed transaction-series numbered (3) may, in symbolic notation, be expressed as follows:

TBal. $\$ 10,000$ || Oral order for $\$ 500 \mid$ Dishonored //

It will be observed that it is concluded by an initial deviational - transaction. That transaction is within the "extinguishment" subdivision of the field of investigation. It is comparable to the sequence which is expressed in symbolic notation as follows:

sBal. $\$ 10,000$ || Counter check for $\$ 500 \mid$ Paid ||

The first comparison is made by substituting for the concluding terms of the transaction the correlative terms of the comparable sequence. The transaction, so modified, and its comparable sequence are, in symbolic notation, expressed as follows:

I A $\left\{\begin{array}{l}\text { TBal. } \$ 10,000 \text { || Oral order for } \$ 500 \mid \text { Paid // } \\ \text { sBal. } \$ 10,000 \text { || Check for } \$ 500 \mid \text { Paid || }\end{array}\right.$

The degree of deviation should be measured in accordance with the rules hereafter stated.

The second comparison is made by substituting for the prior terms of the transaction the correlative terms of the comparable 
sequence. The transaction, so modified, and its comparable sequence are, in symbolic notation, expressed as follows:

I B $\left\{\begin{array}{l}\text { TBal. } \$ 10,000 \text { || Check for } \$ 500 \mid \text { Dishonored // } \\ \text { sBal. } \$ 10,000 \text { || Check for } \$ 500 \mid \text { Paid || }\end{array}\right.$

(4) The assumed transaction-series numbered (4) may, in symbolic notation, be expressed as follows:

TBal. $\$ 10,000$ || Check for $\$ 1,000$ to payee | Payee to his banls |

Presented through clearing-house | Dishonored //

It will be observed that it is concluded by an initial deviational transaction. That transaction is within the "transfer" subdivision of the field of investigation. It is comparable to the sequence which is expressed in symbolic notation as follows:

SBal. $\$ 10,000$ || Check for $\$ 1,000$ to payee | Payee to his banl: | Presented through clearing-house | Paid ||

The first comparison is made by substituting for the concluding terms of the transaction the correlative terms of the comparable sequence. The transaction, so modified, and its comparable sequence are, in symbolic notation, expressed as follows:

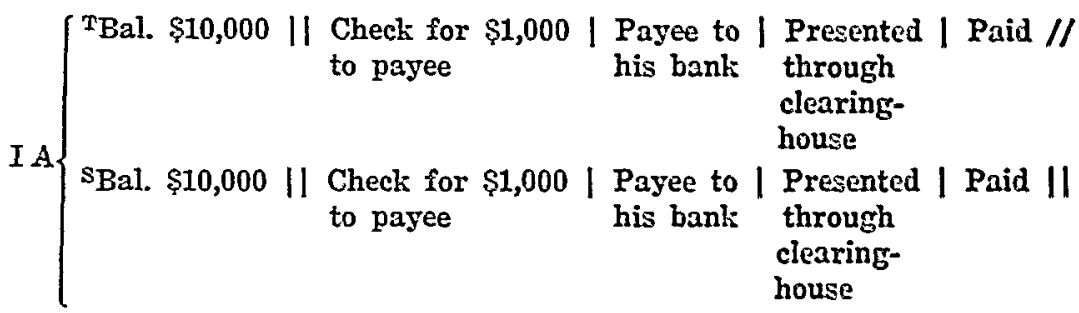

Obviously the terms to be compared are identical. A measurement of the degree of deviation is implicit in this statement.

The second comparison is made by substituting for the prior terms of the transaction the correlative terms of the comparable sequence. The transaction, in this instance, remains unmodified after such substitution. The transaction and its comparable sequence are, in symbolic notation, expressed as follows:

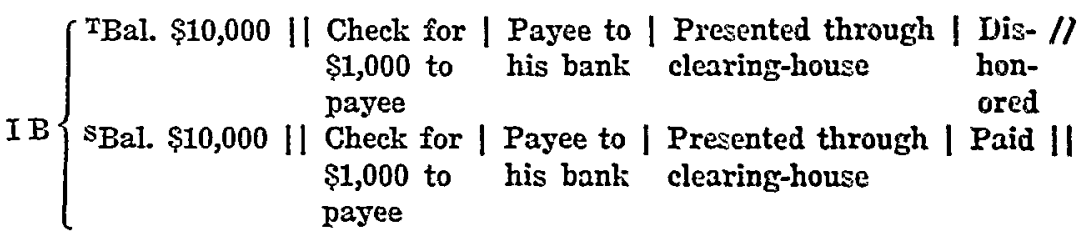

The method proposed for the measurement of degrees of deviation within the field of deposit currency will now be stated. There is observable within this field a high degree of correlation between those courses of behavior which are most profitable and those which are institutional. Profitableness may thus be considered a constant factor in banking institutional transactions. 
There is also, because of the manner in which the abstraction, or institution, was derived, a high degree of correlation between those courses of behavior which frequently are followed and those which are institutional. So that a high frequency of occurrences may be considered also a constant factor in banking institutional transactions. It seems, then, not unreasonable to make the comparison in terms of these two factors: profitableness and familiarity.

Profitableness of a particular transaction is, it will be observed, a complex of four variables: gross return, joint cost, severable cost, losses. Given a perfected statistical method, and ideal conditions for its application, neither the joint cost of a particular transaction, nor in many cases its gross return, can be measured. Since the transaction and its comparable sequence are assumed to have a like conclusion, these factors are equal for each and may be disregarded. It need only be pointed out that since net profit of a particular transaction is the sum rather than the product of these four factors, any ratio composed of only the last two of them (severable cost, losses) for a particular transaction and its comparable sequence will indicate rather than express the ratio between the profitableness of the transaction and the profitableness of the comparable sequence.

A further analysis of these two factors can now be made. A transaction requires a certain expenditure of labor and materials to bring it to a successful conclusion (i.e., to a conclusion conforming to the sequence). This may arbitrarily be called the efficiency of the transaction. This may be derived both for a successful transaction and its comparable sequence. But not all transactions, even those otherwise formally corresponding to a sequence, are in this sense successful. The ratio between the total number of transactions of a particular type (whether sequential or not) and the number of successful transactions of that type may be referred to as the certainty of such transactions. Since the unsuccessful transactions require approximately the same expenditure as the successful transactions, yet bring in no return, their cost must be borne by the successful transactions. Thus the severable cost of a transaction is equal to the product of its efficiency and certainty.

Similarly, what would be expressed as losses when dealing with a number of transactions are expressed as risks when dealing with a single transaction. A risk is simply an expression of the ratio between the number of transactions of a particular type in which a certain sort of loss occurs and the total number of transactions of that type. These risks may be classified in five main types. These can best be indicated by exemplification. Take the simple case of payment of a counter check. If the bank becomes involved in a dispute with its customer it may 
lose the amount of the check. This is called the risk of direct loss. It may have to expend money to prevent loss of the amount of the check. This is called the risk of collection expense. It may lose the good will of the customer or of others through being involved in dispute. This is called the risk of loss of good will. It may in the same way find its credit standing impaired. This is called the risk of loss of credit. Or, finally, if it becomes involved in litigation, it may find the judge and/or jury deciding not in accordance with the facts. This is called the juridical risk.

The risks incurred by the bank have been taken as exemplary. Like risks may be incurred by each actor in a transaction. .

The following table recapitulates the "units" of measurement and is exemplary of the application of the method to the oral order appearing in one of the transaction-series which have been considered. The weighting of the several "units," it should be noted, is hypothetical.

\begin{tabular}{|c|c|c|c|}
\hline & & Oral & Order \\
\hline & $\begin{array}{r}\text { Fac } \\
\text { s } \\
\text { dissi } \\
\text { th } \\
\text { inst }\end{array}$ & $\begin{array}{l}\text { etors of } \\
\text { slight } \\
\text { imilarity } \\
\text { o the } \\
\text { titution }\end{array}$ & $\begin{array}{c}\text { Factors of } \\
\text { gross } \\
\text { dissimilarity } \\
\text { to the } \\
\text { institution }\end{array}$ \\
\hline & Efficiency & & 5 \\
\hline & Certainty & $\mathbf{5}$ & \\
\hline & Familiarity & 5 & \\
\hline & Risk of direct loss & 1 & \\
\hline & Risk of collection expense & 1 & \\
\hline To the bank & Risk of injury to credit & & 1 \\
\hline & Risk of loss of good will & 1 & \\
\hline & Juridical risk & 1 & \\
\hline & Risk of direct loss & 1 & \\
\hline & Risk of collection expense & 1 & \\
\hline To the customer & Risk of injury to credit & & 1 \\
\hline & $\begin{array}{l}\text { Risk of loss of good will } \\
\text { Juridical risk }\end{array}$ & 1 & 1 \\
\hline & & - & \\
\hline & Total & 17 & 8 \\
\hline
\end{tabular}

By the application of the proposed methods of comparison and measurement to "the facts" of a decided case, the correlation between the decision and the measured degree of deviation between "the facts" and the institution can be stated. After the method has been applied to large numbers of cases in many fields it may be possible to state "laws" for some fields in terms of that correlation. Since the institution, if any, may be objectively determined and the degree of deviation between it and the particular transaction-series may be measured approximately, if such "laws" can be stated, their utility in prediction is apparent. 